\title{
Ginsenosides compound (shen-fu) attenuates gastrointestinal injury and inhibits inflammatory response after cardiopulmonary bypass in patients with congenital heart disease
}

\author{
Zhong-yuan Xia, MD, ${ }^{a, b}$ Xian-Yi Liu, MD, ${ }^{a, b}$ Li-ying Zhan, MD, ${ }^{a, b}$ Yi-hong He, BSc, ${ }^{a}$ Tao Luo, MD, ${ }^{a, b}$ \\ and Zhengyuan $\mathrm{Xia}, \mathrm{MD}, \mathrm{PhD}^{\mathrm{a}, \mathrm{b} *}$
}

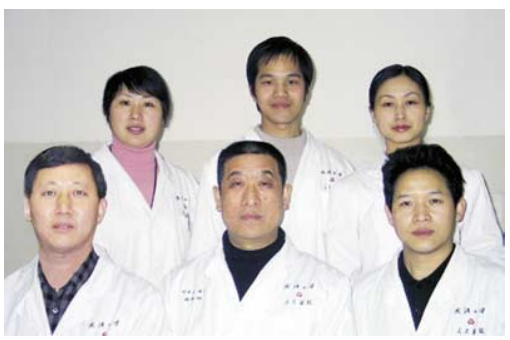

Zhengyuan, Liu, Zhong-yuan (front), Zhan, Luo, He (back)
Objective: This study was undertaken to demonstrate that gastrointestinal mucosal injury occurs during cardiopulmonary bypass in children, increasing systemic inflammatory responses, and to determine whether shen-fu injection (the major components of which are ginsenosides compound, extract of Panax ginseng shown to have antioxidant properties) could attenuate gastrointestinal mucosal injury and subsequent inflammatory responses.

Methods: Twenty-four children undergoing heart surgery for congenital heart defects were randomly assigned to groups $\mathrm{C}$ (placebo control, $\mathrm{n}=12$ ) and $\mathrm{G}(1.35$ $\mathrm{mg} / \mathrm{kg}$ ginsenosides compound intravenously before and throughout the course of cardiopulmonary bypass, $\mathrm{n}=12$ ). Central venous blood samples were taken before cardiopulmonary bypass and at 60 and 120 minutes after aortic declamping (reperfusion). Gastric intramucosal $\mathrm{pH}$ was measured by perioperative tonometry. Plasma lipid peroxidation product malondialdehyde, myocardium-specific creatine kinase isoenzyme MB activity, diamine oxidase, lipopolysaccharide, and interleukin 6 were all measured.

From the Anesthesiology Research Laboratory $^{\mathrm{a}}$ and the Department of Anesthesiology, ${ }^{\text {b }}$ Renmin Hospital, Wuhan University, Wuhan, People's Republic of China.

Presented in part at the First Global Conference on Cardiovascular Clinical Trials and Pharmacotherapy incorporating the Second World Heart Federation Global Conference on Cardiovascular Clinical Trials and Thirteenth International Society of Cardiovascular Pharmacotherapy Congress, Oct 1-3, 2004.

Received for publication Nov 21, 2004; revisions received Jan 26, 2005; accepted for publication Feb 7, 2005.

Address for reprints: Zhengyuan Xia, MD, Anesthesiology Research Laboratory, Department of Anesthesiology, Renmin Hospital, Wuhan University, Wuhan, 430060, People's Republic of China, or Faculty of Pharmaceutical Sciences, The University of British Columbia, $2146 \mathrm{E}$ Mall, Vancouver, BC, V6T 1Z3 Canada (E-mail: zhengyuan_xia@yahoo.com).

*Current affiliation: Division of Pharmacology and Toxicology, Faculty of Pharmaceutical Sciences, The University of British Columbia, Vancouver, British Columbia, Canada.

J Thorac Cardiovasc Surg 2005;130:258-64 $0022-5223 / \$ 30.00$

Copyright () 2005 by The American Association for Thoracic Surgery

doi:10.1016/j.jtcvs.2005.02.046
Results: Significant decrease in gastric intramucosal $\mathrm{pH}$ and increase in plasma diamine oxidase were seen during reperfusion in group $\mathrm{C}$, accompanied by increases in plasma levels of malondialdehyde, lipopolysaccharide, interleukin 6, and creatine kinase isoenzyme MB $(P<.01$ vs before cardiopulmonary bypass). Shen-fu injection significantly attenuated these changes $(P<.05)$. Consequently, fewer patients in group $\mathrm{G}(2 / 12)$ than in group $C(7 / 12)$ needed postoperative inotropic support. Postoperative intensive care unit stay was shorter in group $\mathrm{G}$ than in group C. A tight positive correlation was seen between diamine oxidase and interleukin 6 at 60 minutes after aortic declamping and between diamine oxidase and lipopolysaccharide at 120 minutes after aortic declamping $(r=0.79, P<.0001)$.

Conclusion: Ginsenosides compound may attenuate gastrointestinal injury and inhibit inflammatory response after cardiopulmonary bypass in patients with congenital heart disease.

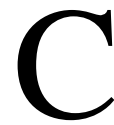
ompromised peripheral perfusion during cardiopulmonary bypass (CPB) and the resulting gastrointestinal mucosal injury leads to a decreased mucosal barrier function, which may allow translocation of intestinal flora and endotoxemia and subsequent increased systemic inflammation. ${ }^{1-3}$ This may lead to or further enhance oxidative stress during $\mathrm{CPB}$, resulting in more eventful postoperative myocardial functional recovery.

Ginsenosides, extracts of Panax ginseng, have been shown to have antioxidant properties. ${ }^{4-6}$ In animal models, ginsenosides, probably primarily the components $\mathrm{Rb}$ and $\mathrm{Ro} 5$, have been shown to protect against myocardial ischemia-reperfusion injury and oxygen free radical production. ${ }^{7,8}$ Ginsenosides are normally divided into two groups according to type of aglycone: the panaxadiol group (eg, Rb1 and Rc) 
and the panaxatriol group (eg, $\operatorname{Rg} 1$ and $\mathrm{Re}$; for detailed structures, see reference list $\left.{ }^{4-9}\right)$. Ginsenosides can undergo transformation by human intestinal bacteria. ${ }^{10,11}$ We postulated that the Chinese traditional medicine ginsenosides compound in shen-fu injection could attenuate myocardial ischemia-reperfusion injury and enhance postoperative myocardial functional recovery in patients undergoing heart operations with CPB. Further, the protective effect of ginsenosides compound might be attributable to its amelioration of gastrointestinal mucosal injury and subsequent systemic inflammatory response or to reduction of oxygen free radical-induced increase in lipid peroxidation.

Studies have shown that endotoxemia is common in children with congenital heart disease and is associated with worse clinical outcomes. ${ }^{12}$ We therefore focused our study on the effects of ginsenosides compound on CPB-induced gastrointestinal injury in children undergoing heart operations for repair of congenital defects. Gastric intramucosal $\mathrm{pH}(\mathrm{pHi})$ and plasma levels of diamine oxidase (DAO) were used as indices of gastric and intestinal mucosal injury, respectively. Lipopolysaccharide (LPS) was used as an index of bacterial endotoxin or endotoxemia.

\section{Patients and Methods}

\section{Patient Selection}

Permission to conduct this study was given by the ethics committee of Renmin Hospital, Wuhan, China. Twenty-four children (cardiac function graded New York Heart Association functional class II or III) with mild to moderate pulmonary hypertension scheduled for operative repair of congenital ventricular septal defect or atrial septal defect were enrolled in this double-blind, randomized study. Primary assessment of pulmonary hypertension was based on clinical, radiographic, and echocardiographic evidence of high pulmonary flow. Absolute pulmonary pressure was measured directly intraoperatively, before CPB. Systemic arterial blood pressure was measured by radial artery catheterization. Patients did not receive either antioxidant vitamins (vitamin C and E) or the cyclooxygenase inhibitor indomethacin before operation.

\section{Anesthesia and Surgical Management}

Anesthesia was induced with intravenous infusion of midazolam, fentanyl, and the muscle relaxant pancuronium and maintained with fentanyl (40-50 $\mu \mathrm{g} / \mathrm{kg}$ ) plus bolus midazolam when appropriate. Patients were ventilated with at fraction of inspired oxygen 1.0 during the procedure. After systemic heparinization, extracorporeal circulation was instituted at a perfusion flow rate of 2.2 to $2.6 \mathrm{~L} /\left(\mathrm{m}^{2} \cdot \mathrm{min}\right)$ with moderate hypothermia $\left(28^{\circ} \mathrm{C}-25^{\circ} \mathrm{C}\right.$ nasopharyngeal temperature). Cardiac asystole was achieved with intermittent multiple-dose cold St Thomas' Hospital cardioplegic solution after continuous application of the aortic crossclamp. Hematocrit was maintained between $22 \%$ and $27 \%$ during and after the operation with packed red blood cells and crystalloid or colloid solution used to supplement circulating blood volume. Dopamine and sodium nitroprusside were given during rewarming until the end of the surgery. Postoperative inotropic support was defined as the use of dopamine with or without concomitant application of epinephrine for 30 minutes or longer during the first 12 postoperative hours. The indication for inotrope administration was mean radial arterial blood pressure less than $60 \mathrm{~mm} \mathrm{Hg}$. For a better comparison between study groups, absolute doses of dopamine were recorded. All operations were performed by the same surgical team, and physicians working in the operating room and the intensive care unit were blinded to treatment protocols. The decisions for extubation and discharge from the intensive care unit were made according to preset criteria.

\section{Experimental Protocol}

Eligible patients were randomly allocated to two groups after anesthesia induction and before surgery: group $\mathrm{C}$ (control, $\mathrm{n}=12$ ) and group $\mathrm{G}$ (ginsenosides treatment, $\mathrm{n}=12$ ).

Shen-fu injection (Sanjiu Medicine Company, Shenzhen, China) contains $0.9 \mathrm{mg}$ ginsenosides and $0.1 \mathrm{mg}$ aconite alkaloid per milliliter. It was injected intravenously into patients in group $\mathrm{G}$ at a dosage of $0.5 \mathrm{~mL} / \mathrm{kg} 2$ minutes before the start of CPB and completed in 5 minutes. This was followed by a continuous infusion of shen-fu at a dosage of $1.0 \mathrm{~mL} / \mathrm{kg}$ throughout the course of $\mathrm{CPB}$. For continuous infusion, shen-fu was diluted in double volumes of saline solution (1:2 volume/volume dilution), and the diluted solution was infused at the rate of $0.04 \mathrm{~mL} /(\mathrm{kg} \cdot \min )$ by a Grasby pump. The total dosage of shen-fu applied to group $\mathrm{G}$ patients was $1.5 \mathrm{~mL} / \mathrm{kg}$ (equal to $1.35 \mathrm{mg} / \mathrm{kg}$ ginsenosides and 0.15 $\mathrm{mg} / \mathrm{kg}$ aconite alkaloid). Patients in group $\mathrm{C}$ received equal volumes of saline solution placebo.

Blood samples were taken from a central venous cannulation at three time points: after anesthesia induction and before CPB (preCPB), 60 minutes after aortic declamping (reperfusion 60), and 120 minutes after aortic declamping (reperfusion 120). Gastric pHi was measured by perioperative tonometry.

Blood samples were collected in duplicate. One part of each blood sample was processed on the same day for myocardiumspecific creatine kinase MB (CK-MB) activities and the lipid peroxidation product malondiadehyde (MDA) content. Another part was immediately centrifuged, and the plasma was frozen at $-70^{\circ} \mathrm{C}$ and stored until assay for LPS, interleukin 6 (IL-6), and DAO.

\section{Bioassays}

Plasma MDA and CK-MB were measured by chemical analysis with commercial kits (Nanjing Jiangzheng Biological Engine Institute, Nanjing, China) as previously described. ${ }^{13}$ Values of LPS, expressed as endotoxin units per milliliter of plasma $(\mathrm{EU} / \mathrm{mL})$, was measured by chromogenic Limulus amebocyte lysate as described by Saraf and colleagues. ${ }^{14}$ Plasma DAO was measured with the method described by Klocker and coworkers, ${ }^{15}$ and IL-6 was detected with chemiluminescent enzyme immunoassay commercial kits (Fujirebio, Tokyo, Japan). All biochemical assays were performed in duplicate. The measurements of LPS, DAO, and IL-6 were performed in the laboratory of the Trauma Surgery Institution of 304 Hospital of People's Liberation Army, Beijing, China. Samples were coded, and the laboratory investigator was blinded with regard to the study groups. 
TABLE 1. Patient profile and perioperative data

\begin{tabular}{lcc}
\hline & Group C (n= 12) & Group G (n = 12) \\
\hline Age (y) & & $12.6 \pm 0.8$ \\
$\quad$ Mean \pm SEM & $12.9 \pm 0.6$ & $(8-15)$ \\
$\quad$ Range & $(10-15)$ & $6: 6$ \\
Sex (male/female) & $5: 7$ & $40.0 \pm 3.6$ \\
Weight (kg, mean \pm SEM) & $39.6 \pm 3.1$ & $10: 2$ \\
Congenital heart defect (VSD/ASD) & $11: 1$ & $75.6 \pm 4.0$ \\
CPB time (min, mean \pm SEM) & $77.2 \pm 4.3$ & $41.2 \pm 2.5$ \\
Aortic crossclamp time (min, mean \pm SEM) & $44.4 \pm 2.7$ & $2: 10^{*}$ \\
Post-CPB inotropes (yes/no) & $7: 5$ & $16.8 \pm 2.2^{*}$ \\
Dose of dopamine used (mg/kg, mean \pm SEM) & $32.9 \pm 3.3$ & $6.3 \pm 0.2^{*}$ \\
Postoperative extubation (h, mean \pm SEM) & $10.8 \pm 0.7$ & $2.4 \pm 0.2^{*}$ \\
Postoperative ICU stay (d, mean \pm SEM) & $3.3 \pm 0.2$ & $13.2 \pm 0.2$ \\
Postoperative hospital stay (d, mean \pm SEM) & $13.9 \pm 0.3$ & \\
\hline
\end{tabular}

$V S D$, Ventricular septal defect; $A S D$, atrial septal defect; $C P B$, cardiopulmonary bypass; $I C U$, intensive care unit. $* P<.05$ versus group $C\left(\chi^{2}\right.$ test or Student $t$ test).

TABLE 2. Perioperative hemodynamic data

\begin{tabular}{|c|c|c|c|}
\hline & Preoperative & Reperfusion 60 & Reperfusion 120 \\
\hline \multicolumn{4}{|c|}{$\mathrm{SBP}(\mathrm{mm} \mathrm{Hg})$} \\
\hline Group C & $105.8 \pm 3.4$ & $89.7 \pm 1.8^{*}$ & $95.7 \pm 2.3^{*}$ \\
\hline Group G & $110.2 \pm 3.4$ & $95.3 \pm 2.3^{*} \dagger$ & $106.7 \pm 2.8 \dagger$ \\
\hline \multicolumn{4}{|c|}{$\mathrm{DBP}(\mathrm{mm} \mathrm{Hg})$} \\
\hline Group C & $68.9 \pm 2.2$ & $55.7 \pm 2.1^{*}$ & $59.9 \pm 2.1^{*}$ \\
\hline Group G & $70.7 \pm 2.7$ & $61.7 \pm 2.0^{*} \dagger$ & $69.0 \pm 2.4 \dagger$ \\
\hline \multicolumn{4}{|c|}{$\mathrm{MAP}(\mathrm{mm} \mathrm{Hg})$} \\
\hline Group C & $81.2 \pm 2.5$ & $67.1 \pm 1.9^{*}$ & $71.8 \pm 2.1^{*}$ \\
\hline Group G & $84.2 \pm 2.9$ & $72.9 \pm 2.0^{*} \dagger$ & $81.9 \pm 2.5 \dagger$ \\
\hline \multicolumn{4}{|c|}{ Heart rate (beats/min) } \\
\hline Group C & $89 \pm 3$ & $102 \pm 3^{*}$ & $96 \pm 3$ \\
\hline Group G & $87 \pm 2$ & $93 \pm 2.0 \dagger$ & $88 \pm 2 \dagger$ \\
\hline \multicolumn{4}{|c|}{ Rate-pressure product } \\
\hline Group C & $9449 \pm 469$ & $9158 \pm 309$ & $9192 \pm 301$ \\
\hline Group G & $9628 \pm 258$ & $8812 \pm 259^{*}$ & $9377 \pm 257$ \\
\hline \multicolumn{4}{|c|}{ Mean PAP $(\mathrm{mm} \mathrm{Hg})$} \\
\hline Group C & $31.5 \pm 1.6$ & - & - \\
\hline Group G & $33.3 \pm 1.4$ & - & - \\
\hline
\end{tabular}

All data are mean \pm SEM $(n=12$ per group). $S B P$, Systolic blood pressure; $D B P$, diastolic blood pressure; $M A P$, mean arterial pressure; $P A P$, pulmonary artery pressure. $* P<.05$ versus preoperative value. $\dagger P<.05$ versus group $\mathrm{C}$.

\section{Statistical Analysis}

All continuous data were expressed as mean \pm SEM. Statistical evaluation of patient files and perioperative data were performed by unpaired Student $t$ test or $\chi^{2}$ test as appropriate. Between-group and within-group differences of bioassay data were analyzed with 2-way analysis of variance with repeated measures and Bonferroni corrections (Prism; GraphPad Software, Inc, San Diego, Calif) as appropriate. Correlations were evaluated by the Pearson test.

\section{Results}

Patient Profile and Perioperative Data

Patient demographic and perioperative data are presented in Tables 1 and 2. Patients were similar in terms of age, body weight, diagnosis, preoperative systemic and pulmonary arterial pressures, durations of CPB and aortic crossclamping, and overall postoperative hospital stay. Dosages of heparin were also similar between groups. After aortic declamping, all patients were transiently (for less than 5-10 minutes) given dopamine to assist circulation. Seven patients in group $\mathrm{C}(7 / 12)$ needed sustained postoperative inotropic support, versus 2 patients in group $\mathrm{G}(2 / 12, P<$ $.05)$. One patient in group $\mathrm{C}$ needed concomitant application of dopamine and epinephrine. There was no echocardiographic evidence of residual defect in patients who needed postoperative inotropic support in either group. The post- 
operative durations of mechanical ventilation $(6.3 \pm 0.2$ hours in group $\mathrm{G}$ vs $10.8 \pm 0.7$ hours in group $\mathrm{C}, P<.01$ ) and intensive care unit stay were significantly shorter in group $\mathrm{G}$ than in group C. Most patients were discharged within 2 postoperative weeks.

\section{Post-CPB Hemodynamic Changes}

As shown in Table 2, at reperfusion 60, systolic blood pressure (SBP), diastolic blood pressure (DBP), and mean arterial pressure (MAP) were lower than the corresponding pre-CPB values in both groups $(P<.05)$. This change was accompanied by a significant increase in heart rate in group $\mathrm{C}$ but not in group G. At reperfusion 120, SBP, DBP, and MAP in group $\mathrm{G}$ (but not in group $\mathrm{C}$ ) returned to pre-CPB levels and were significantly higher than those in group $\mathrm{C}$. Notably, at reperfusion 60, the rate (heart rate) and pressure (SBP) product, an indirect index of myocardial oxygen demand, was significantly decreased in group $\mathrm{G}$, but not in group C relative to pre-CPB values. Still, blood pressures in group $\mathrm{G}$ were significantly higher than those in group $\mathrm{C}$ at reperfusion 60 .

\section{Plasma Levels of MDA and CK-MB}

Preoperative levels of MDA (Figure 1, A) did not differ between groups. MDA in group $\mathrm{C}$ but not in group $\mathrm{G}$ increased significantly during reperfusion relative to preCPB levels. Levels of CK-MB were significantly enhanced during reperfusion in both groups relative to pre-CPB values, but the values were lower in group $\mathrm{G}$ than in group $\mathrm{C}$ at reperfusion 60 and reperfusion $120(P<.05$ or 0.01$)$.

\section{Gastric pHi and Plasma Levels of DAO, LPS, and IL-6}

Gastric pHi values in group C (mean \pm SEM $7.37 \pm 0.01$ ) and group $\mathrm{G}(7.36 \pm 0.01)$ were not different at pre-CPB (Figure 1, A). Values decreased to $7.17 \pm 0.02$ at reperfusion 60 and $7.20 \pm 0.01$ at reperfusion 120 in group $C(P<$ .001 vs pre-CPB) and were significantly lower than the corresponding values in group $\mathrm{G}(7.30 \pm 0.01$ at reperfusion 60 and $7.32 \pm 0.01$ at reperfusion $120, P<.001$ vs group C). Levels of plasma DAO (Figure 2, $B$ ) were significantly increased at reperfusion 60 and maintained elevated at reperfusion 120 in both groups $(P<.01$ or $P<.05$ vs pre-CPB). DAO levels at reperfusion 60 and reperfusion 120 in group $\mathrm{G}$ were lower than those in group $\mathrm{C}(P<.05$ and $P<.01)$. During reperfusion, the increases in plasma levels of LPS (Figure 2, C) and IL-6 (Figure 2,D) in group $\mathrm{C}$ mirrored the changes of plasma DAO. Treatment with the ginsenosides compound shen-fu attenuated the significant increases of LPS and IL-6 seen in group C.

\section{Correlation Analysis}

As shown in Figure 3, an inverse correlation between gastric pHi and plasma DAO $(r=-0.73, P<.0001)$ and a positive correlation between plasma DAO and LPS $(r=$
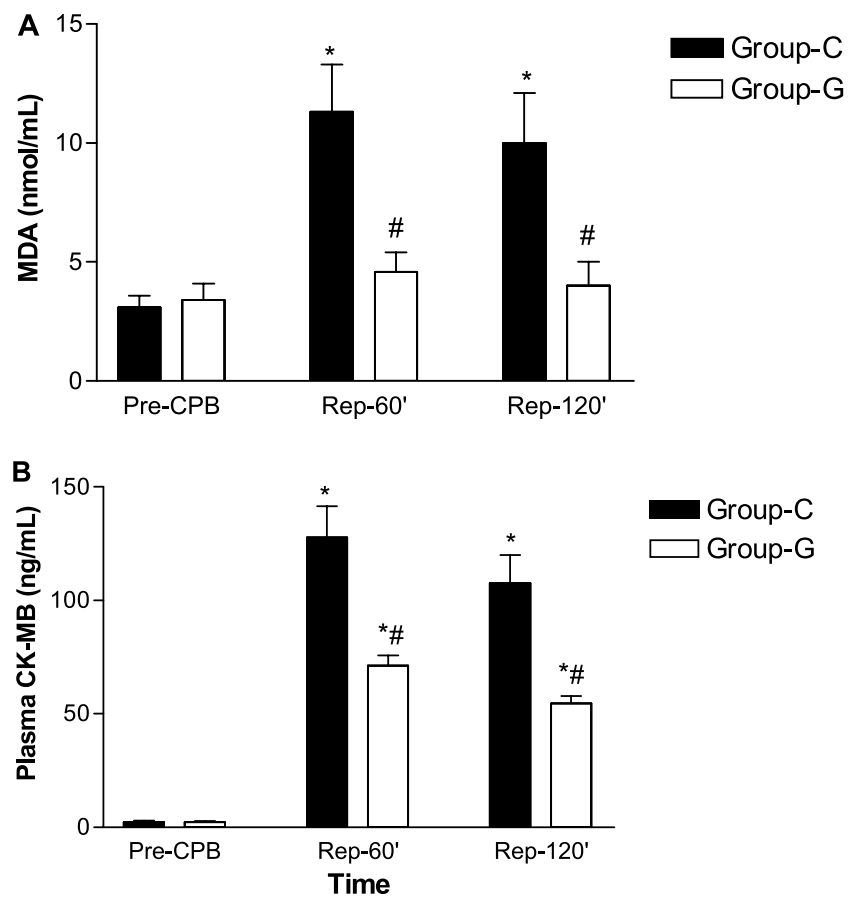

Figure 1. Variations in perioperative plasma levels of MDA (A) CK-MB (B). Rep-60', Reperfusion 60; Rep-120', reperfusion 120. Asterisk indicates $P<.05$ or $P<.01$ vs pre-CPB; crosshatch indicates $P<.05$ or $P<.01$ vs group $C$ ).

$0.79, P<.0001)$ were observed at reperfusion 120 . Also, a tight positive correlation was seen between plasma LPS and IL-6 levels at reperfusion $60(r=0.64, P=.0007)$. However, a correlation between gastric pHi and plasma LPS or IL-6 was not detected. Figure 4 depicts an overall tight positive correlation between plasma levels of DAO and IL-6 at reperfusion $60(r=0.79, \mathrm{n}=24, P<.0001)$, although this relationship is largely a reflection of the correlation between DAO and LPS in group $\mathrm{C}(r=0.77, \mathrm{n}=12, P=$ $.004)$.

\section{Discussion}

Our study is the first to investigate the effects of a novel therapeutic approach on the relationship of gastrointestinal tract injury, systemic inflammatory response, and postoperative myocardial functional recovery after cardiac surgery in patients with congenital heart disease. The major findings include the following: (1) Gastrointestinal tract injury is apparent in children undergoing heart operations with CPB, as evidenced by significant decrease of gastric pHi and increase in plasma DAO, accompanied by elevated plasma levels of LPS and IL-6 and post-CPB myocardial cellular damage. (2) The tight correlations detected between plasma levels of DAO and LPS as well as IL-6 suggest intestinal mucosal injury as a potential cause of elevated systemic 

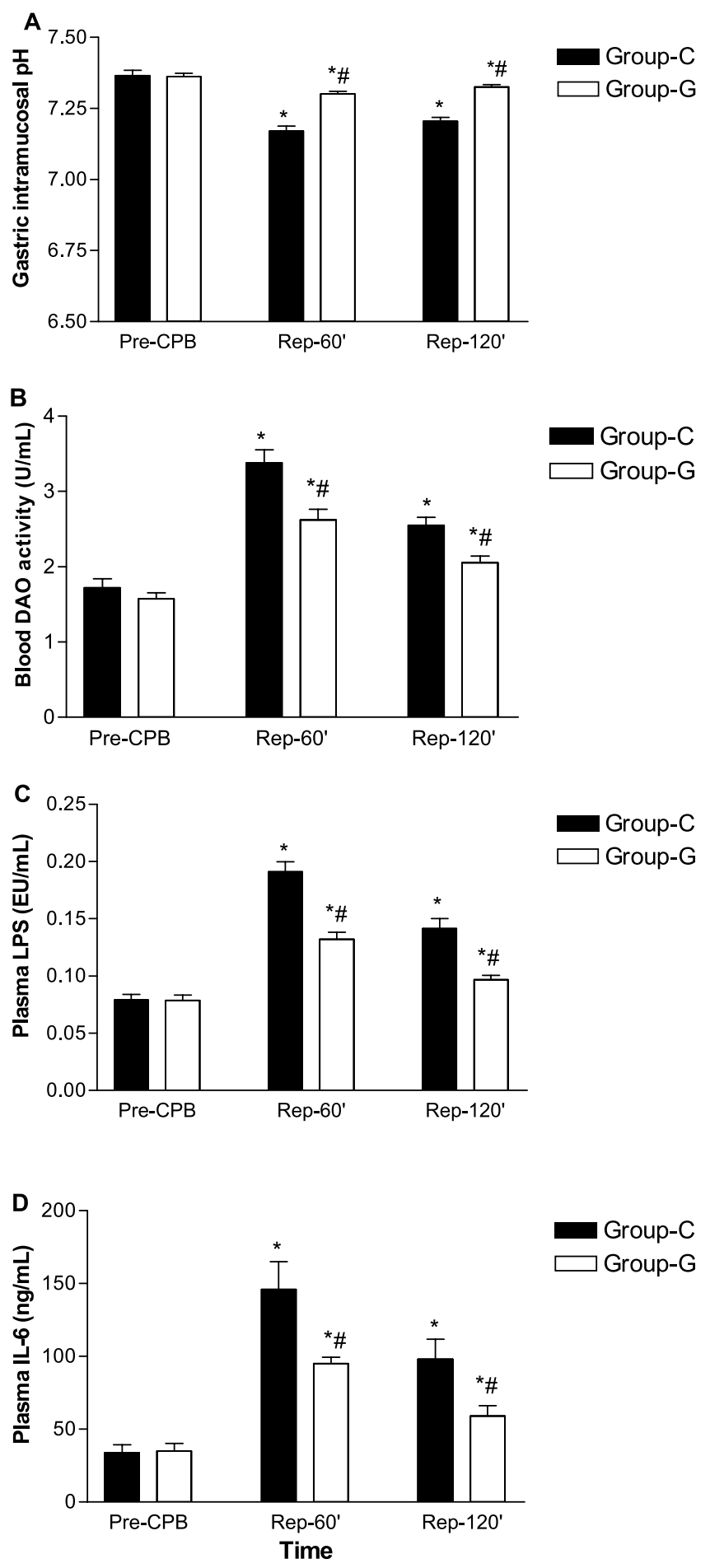

Figure 2. Variations of perioperative gastric $\mathrm{pHi}(\mathrm{A})$ and plasma levels of DAO (B), LPS (C), and IL-6 (D). Rep-60', Reperfusion 60; Rep-120', reperfusion 120. Asterisk indicates $P<.05$ or $P<.01$ vs pre-CPB; crosshatch indicates $P<.05$ or $P<.01$ vs group $C$ ). endotoxemia and inflammatory response during CPB, as least in children.

$\mathrm{DAO}$, formerly called histaminase, is found in various tissues but is especially active in the intestinal mucosa. Its function is the oxidative deamination of several polyamines, essential substances for cell proliferation. DAO is thus a regulatory enzyme in rapidly proliferating tissues such as intestinal mucosa. DAO is normally present in very small amounts in the circulation, and its basal plasma levels are positively correlated with the maturity and integrity of the intestinal mucosa. Circulating DAO has been documented to be elevated in intestinal ischemia. ${ }^{25}$ Measurement of plasma DAO activity can therefore be used as an index of intestinal mucosal ischemia. Recently, it has been reported that serum DAO is increased in patients undergoing coronary artery bypass grafting with $\mathrm{CPB}$ and is accompanied by systemic endotoxemia. ${ }^{16}$ Our study confirms and extends the findings of Tsunooka and colleagues ${ }^{16}$ in children. Further, the results from our study suggest that intestinal mucosal injury not only may be a cause of systemic endotoxemia, as evidenced by the increase in plasma levels of LPS, but also may be responsible for the increase in systemic cytokines such as IL-6, as measured in this study. The elevations of plasma LPS and IL-6 mirrored the increase of plasma DAO in group $\mathrm{C}$ and group $\mathrm{G}$. This may suggest that treatments designed to prevent intestinal mucosal injury during $\mathrm{CPB}$ could also be a good choice of therapy in an effort to attenuate systemic inflammatory responses.

Ginsenosides compound significantly reduced plasma levels of MDA, a commonly used index of lipid peroxidation, during reperfusion (Figure 1, A). This should have contributed in part to the attenuated myocardial cellular damage and facilitated postoperative myocardial functional recovery seen in group G. Oxygen free radical-induced lipid peroxidation has been reported to adversely influence postoperative myocardial functional recovery in patients undergoing cardiac surgery with CPB.${ }^{17}$ Ginsenosides compound's antioxidant property may also be a factor leading to the attenuation of gastrointestinal injury observed in group G. Experimental studies have shown that oxygen free radicals exacerbate gastrointestinal mucosal ischemia-reperfusion injury and that this damage is preventable by oxygen free radical scavengers. ${ }^{18,19}$

A statistically significant correlation between gastric intramucosal acidosis (decrease in values of $\mathrm{pHi}$ ), a reflection of gastric intramucosal ischemic injury, and plasma DAO suggests that during $\mathrm{CPB}$ gastric and intestinal mucosal ischemic injury occur concomitantly. Consequently, measurement of plasma DAO might be used as surrogate of gastric $\mathrm{pHi}$, at least in the current clinical setting. Given the large surface area of the intestinal mucosa, because of the extensive network of the villi, intestinal mucosal injury during $\mathrm{CPB}$ may have a more significant impact than gastric 

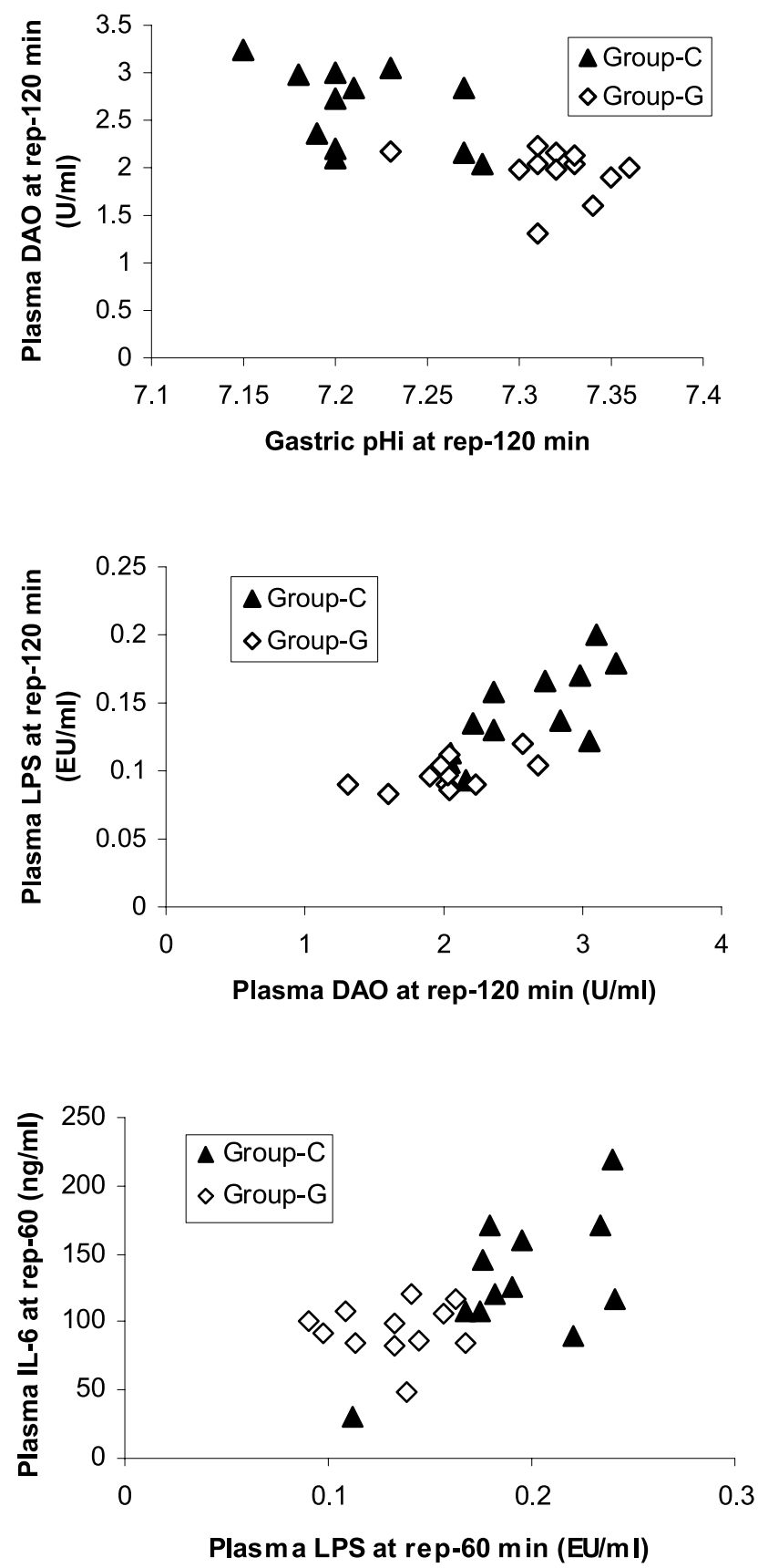

Figure 3. Relationship between plasma gastric $\mathrm{pHi}$ and $\mathrm{DAO}$ at reperfusion 120 min (rep-120; top), between plasma DAO and LPS at reperfusion 120 (middle), and between plasma LPS and IL-6 at reperfusion 60 (rep-60; bottom) in groups C (solid triangles) and G (open diamonds). Inverse correlation between gastric $\mathrm{pHi}$ and plasma DAO ( $r=-0.73,95 \%$ confidence interval -0.9749 to $-0.4605, P<.0001)$ and positive correlation between plasma DAO and LPS ( $r=0.79,95 \%$ confidence interval 0.5662-0.9447, $P$ $<.0001$ ) were observed at reperfusion 120. Also, tight positive correlation is seen between plasma LPS and IL-6 at reperfusion $60(r=0.64,95 \%$ confidence interval $0.3232-0.8308, P=.0007)$.

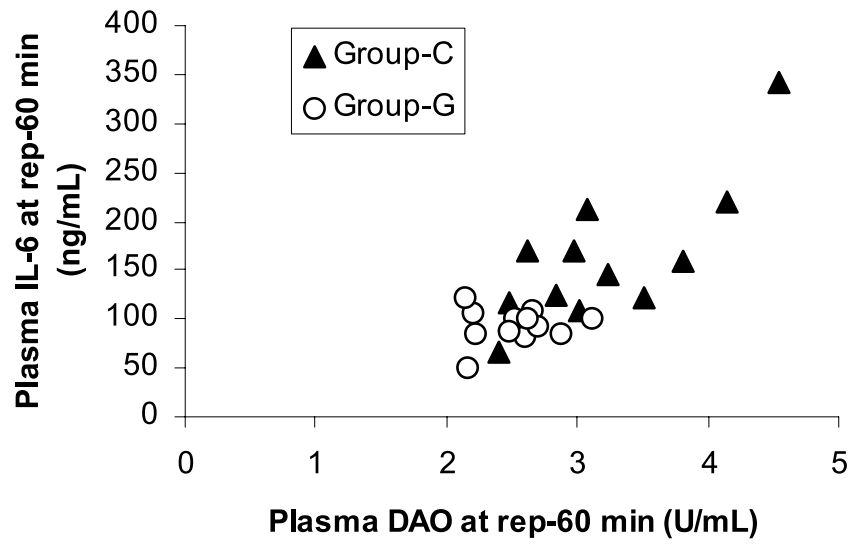

Figure 4. Relationship between plasma levels of IL-6 and DAO at reperfusion 60 (rep-60) in groups C (solid triangles) and G (open diamonds). Overall tight positive correlation is seen between DAO and IL-6 $(r=0.79,95 \%$ confidence interval 0.5758-0.9073, $n=$ $24, P<.0001)$. This relationship largely reflects correlation between DAO and LPS in group $C(r=0.77,95 \%$ confidence interval $0.3490-0.9317, \mathrm{n}=12, P=.004)$. No such relationship is seen in group $\mathbf{G}(P=.6)$.

mucosal injury on systemic endotoxemia and increase of inflammatory response seen in this study.

IL-6 is generally considered a nonspecific marker of inflammation. Its formation is increased with endotoxemia and after CPB. ${ }^{18,19}$ Increased plasma levels of IL- 6 have been reported to be associated with compromised postoperative myocardial functional recovery in patients undergoing cardiac surgery with $\mathrm{CPB} .{ }^{20}$ In our study, treatment with ginsenosides compound reduced postoperative IL-6, attenuated postoperative myocardial cellular damage (as evidenced by significant reduction in plasma levels of CK$\mathrm{MB}$ ), and facilitated myocardial functional recovery. This might be a consequence of attenuated gastrointestinal injury and endotoxemia, because circulating endotoxin is a main factor triggering inflammatory responses.

Heparin is known to cause immediate rise in plasma DAO activity that peaks within 30 to 60 minutes after heparin injection, decreasing gradually after that. ${ }^{24}$ In our study, blood was sampled 60 and 120 minutes after aortic declamping. This should have minimized, if not avoided, the potential confounding factor of heparin effect on DAO release.

It should be noted that although the major component of shen-fu used this study is ginsenosides compound, aconite alkaloid (another component of shen-fu) has also been shown to be protective against myocardial ischemia-reperfusion injury. ${ }^{21}$ Shen-fu is more cardioprotective than its separate components ginsenosides compound and aconite alkaloid. ${ }^{21}$ It is also noteworthy that the rate-pressure product (an index of myocardial oxygen demand) in group $\mathrm{G}$ but not in group 
$\mathrm{C}$ was significantly reduced at reperfusion 60 relative to its pre-CPB values. Patients in group G maintained high levels of blood pressure (SBP, DBP, and MAP) relative to group $\mathrm{C}$ at reperfusion 60 . Ginsenosides have been shown to stimulate nitric oxide release. ${ }^{7}$ This is a potential mechanism whereby shen-fu increased myocardial oxygen use efficiency during early reperfusion and deserves further study. A recent study shows that nitric oxide contributes to oxygen demand-supply balance in hypoperfused myocardium. ${ }^{22}$

In summary, results from our study indicate that gastrointestinal injury and endotoxemia are common in children undergoing heart operations to correct congenital heart disease. Shen-fu, the major component of which is ginsenosides compound, inhibits inflammatory response after cardiopulmonary bypass in patients with congenital heart disease, which should favor postoperative myocardial functional recovery. The beneficial effects of shen-fu may be attributable in part to its effects in alleviating the intestinal mucosal epithelial cell injuries and cytokine release normally seen with ischemia-reperfusion. ${ }^{23}$ The mechanisms of shen-fu's cardioprotection remain to be elucidated.

We are grateful to Dr Yao Yongming, Trauma Surgery Central Laboratory of 304th Hospital of PLA, Beijing, China, for his help in performing bioassays for LPS, IL-6, and DAO.

\section{References}

1. Mollhoff T, Loick HM, Van Aken H, Schmidt C, Rolf N, Tjan TD, et al. Milrinone modulates endotoxemia, systemic inflammation, and subsequent acute phase response after cardiopulmonary bypass (CPB). Anesthesiology. 1999;90:72-80.

2. Riddington DW, Venkatesh B, Boivin CM, Bonser RS, Elliott TS, Marshall T, et al. Intestinal permeability, gastric intramucosal $\mathrm{pH}$, and systemic endotoxemia in patients undergoing cardiopulmonary bypass. JAMA. 1996;275:1007-12.

3. Watarida S, Mori A, Onoe M, Tabata R, Shiraishi S, Sugita T, et al. A clinical study on the effects of pulsatile cardiopulmonary bypass on the blood endotoxin levels. J Thorac Cardiovasc Surg. 1994;108:620-5.

4. Liu ZQ, Luo XY, Liu GZ, Chen YP, Wang ZC, Sun YX. In vitro study of the relationship between the structure of ginsenoside and its antioxidative or prooxidative activity in free radical induced hemolysis of human erythrocytes. J Agric Food Chem. 2003;51:2555-8.

5. Chen X, Li YJ, Deng HW, Yang BC, Li DY, Shen N. Protective effects of ginsenosides on anoxia/reoxygenation of cultured rat myocytes and on reperfusion injuries against lipid peroxidation. Biomed Biochim Acta. 1987;46:S646-9.

6. Yokozawa T, Satoh A, Cho EJ. Ginsenoside-Rd attenuates oxidative damage related to aging in senescence-accelerated mice. $J$ Pharm Pharmacol. 2004;56:107-13.

7. Chen X. Cardiovascular protection by ginsenosides and their nitric oxide releasing action. Clin Exp Pharmacol Physiol. 1996;23:728-32.

8. Chu GX, Chen X. Anti-lipid peroxidation and protection of ginsenosides against cerebral ischemia-reperfusion injuries in rats. Zhongguo Yao Li Хие Bao. 1990;11:119-23.
9. Scott GI, Colligan PB, Ren BH, Ren J. Ginsenosides Rb1 and Re decrease cardiac contraction in adult rat ventricular myocytes: role of nitric oxide. Br J Pharmacol. 2001;134:1159-65.

10. Bae EA, Han MJ, Kim EJ, Kim DH. Transformation of ginseng saponins to ginsenoside $\mathrm{Rh} 2$ by acids and human intestinal bacteria and biological activities of their transformants. Arch Pharm Res. 2004;27:61-7.

11. Bae EA, Park SY, Kim DH. Constitutive beta-glucosidases hydrolyzing ginsenoside $\mathrm{Rb} 1$ and $\mathrm{Rb} 2$ from human intestinal bacteria. Biol Pharm Bull. 2000;23:1481-5.

12. Lequier LL, Nikaidoh H, Leonard SR, et al. Preoperative and postoperative endotoxemia in children with congenital heart disease. Chest. 2000;117:1706-12.

13. Xia Z, Gu J, Ansley DM, Xia F, Yu J. Antioxidant therapy with Salvia miltiorrhiza decreases plasma endothelin- 1 and thromboxane $B_{2}$ after cardiopulmonary bypass in patients with congenital heart disease. J Thorac Cardiovasc Surg. 2003;126:1404-10.

14. Saraf A, Larsson L, Burge H, Milton D. Quantification of ergosterol and 3-hydroxy fatty acids in settled house dust by gas chromatography-mass spectrometry: comparison with fungal culture and determination of endotoxin by a Limulus amebocyte lysate assay. Appl Environ Microbiol. 1997;63:2554-9.

15. Klocker J, Perkmann R, Klein-Weigel P, Morsdorf G, Drasche A, Klingler A, et al. Continuous administration of heparin in patients with deep vein thrombosis can increase plasma levels of diamine oxidase. Vascul Pharmacol. 2004;40:293-300.

16. Tsunooka N, Maeyama K, Hamada Y, Imagawa H, Takano S, Watanabe Y, et al. Bacterial translocation secondary to small intestinal mucosal ischemia during cardiopulmonary bypass. Measurement by diamine oxidase and peptidoglycan. Eur J Cardiothorac Surg. 2004; 25:275-80.

17. Ansley DM, Xia Z, Dhaliwal BS. The relationship between plasma free 15-F2t-isoprostane concentration and early postoperative cardiac depression following warm heart surgery. J Thorac Cardiovasc Surg. 2003; $126: 1222-3$

18. Tomatsuri N, Yoshida N, Takagi T, Katada K, Isozaki Y, Imamoto E, et al. Edaravone, a newly developed radical scavenger, protects against ischemia-reperfusion injury of the small intestine in rats. Int $\mathrm{J} \mathrm{Mol}$ Med. 2004;13:105-9.

19. Cabeza J, Motilva V, Martin MJ, de la Lastra CA. Mechanisms involved in gastric protection of melatonin against oxidant stress by ischemia-reperfusion in rats. Life Sci. 2001;68:1405-15.

20. Liebold A, Langhammer T, Brunger F, Birnbaum DE. Cardiac interleukin-6 release and myocardial recovery after aortic crossclamping. Crystalloid versus blood cardioplegia. J Cardiovasc Surg (Torino). 1999;40:633-6.

21. Zheng SY, Sun J, Zhao X, Xu JG. Protective effect of shen-fu on myocardial ischemia-reperfusion injury in rats. Am J Chin Med. 2004; 32:209-20.

22. Setty S, Tune JD, Downey HF. Nitric oxide contributes to oxygen demand-supply balance in hypoperfused right ventricle. Cardiovasc Res. 2004;64:431-6.

23. Xia ZY, Meng QT, Zhang F, Chen XD. Effect of shenfu injection (ginesenoside and aconite alkaloid) on the apoptosis of intestinal mucosal epithelial cells and its mechanism during ischemia-reperfusion in rats. Chin J Traumatol. 2004;7:363-7.

24. Rokkas T, Vaja S, Murphy GM, Dowling RH. Postheparin plasma diamine oxidase in health and intestinal disease. Gastroenterology. 1990;98:1493-501.

25. Bragg LE, Thompson JS, West WW. Intestinal diamine oxidase levels reflect ischemic injury. J Surg Res. 1991;50:228-33. 